\title{
Robotic Search and Rescue using Human Detection System
}

\author{
Golande Avinash L ${ }^{1}$, Mali Shivam Jalindar ${ }^{2}$, Mitkari Nikhil Ajay ${ }^{3}$ \\ ${ }^{1}$ SPM's Rajarshi Shahu College of Engineering, Faculty, \\ 2,3 JSPM's Rajarshi Shahu College of Engineering, Student,
}

Article History: Received: 10 November 2020; Revised: 12 January 2021; Accepted: 27 January 2021; Published online: 05 April 2021

\begin{abstract}
In this Technological advancement period, advanced construction improvements lead the formation of skyscrapers and homes which expanded the dangers of losing life because of natural and manmade catastrophes. In this system, we are proposing a radio-controlled bot that can identify live human beings from which are in the inaccessible region. Python libraries are used in Raspberry Pi microcontroller having Camera module to catch pictures of objects around it. This paper discusses about the mentioned system. The project takes live image samples and sends it to a network where this images can be accessed through a device. This images can be used for human detection. PIR sensor is used for the detection of human being trapped under debris. Whenever a human is detected the bot will send GPS co-ordinates to the device.
\end{abstract}

Keywords: Raspberry Pi (RPi), Human Detection, Live Image Processing, PIR, Single Board Computer (SBC), Radio controlled bot.

\section{Introduction}

In today's world lots of natural calamities are occurring resulting in life loss. We can't prevent the natural calamities to occur but can prevent the people affecting from it. Natural calamities bring a large amount of destruction with it lowering the response time to search and find the survivors. At times it becomes difficult for rescue team to go in each and every place and rescue humans. Rescue operations always take too much time. This whole scenario creates an opportunity to develop system which can locate survivors by sensing them. Here the rescue robot can play a key role as it is controlled by human using a radio control. The bot can send live images to device as well as can detect the human trapped under debris.

The most outstanding work on search and rescue robots in the US has been done by R. Murphy and collaborators, specifically on the utilization of many radio operated robots for the rescue and search. In collaboration with expert professionals, incorporating the interest in the rescue activities of the World Trade Center (WTC), later in September 11 assaults A genuine situation which imitates a few true circumstances looked by human groups after earthquake situations, which has been broadly utilized around the world, e.g., in Europe, at the Intelligent Frameworks for Emergencies and Civil Defence in Rome, Italy, also it has been filling in as a testbed.

Robo Cup rescue activity National Institute of Standards and Technology has additionally built up the USAR Performance Metrics and Test Arena.

Which combines every year many groups in an inquiry and salvage automated challenge. This solid overall enthusiasm for hunt and salvage robot Innovative work has pulled in a few organizations that have created USAR business stages, for example, iRobot (Pack bot, an extremely powerful, light and portable tele-worked robot, utilized in the WTC tasks. Other than that as of late re-focused on towards military tasks), Robo Probe Tech (bomb in capacitating and review robots), Inuktun (tele-worked robots likewise utilized in the WTC tasks).

Innovative work has pulled in a few organizations that have created USAR business stages, for example, iRobot (Packbot, an extremely powerful, light and portable tele- worked robot, utilized in the WTC tasks however as of late re-focused on towards military tasks), Robo Probe Technologies (bomb incapacitating and review robots), Inuktun (tele-worked robots likewise utilized in the WTC tasks).

The paper is sorted out as follows: Section II portrays the system importance of the robot and the plan limitations forced by the conditions where the robot should work. Section III contains detecting capacities and the blended fastened/remote correspondence framework, the most creative component of the bot, are depicted in. Section IV covers the working principle piece of this tele operated robot. A portrayal of a few tests made which were very sensible situations is made in Section V. Section VII close the paper, reaching primary determinations and posting 
points of great future work with regards to the robot. References are described in Section VIII.

\section{Related Work}

The system proposes a versatile robot which moves in the catastrophe, quake inclined area and aides in recognizing the live people also the disaster affected people live people's, area and send rescue forces. Thus owing to the on convenient finding in natural catastrophes this can spare valuable life and incredible misfortune yet lacking the assistance of an enormous numeral of rescue teams.

[2]Using camera feed bot was controlled and directed by using raspberry pi once the human is detected alert is given have used only image detection algorithm

[14] Successful route finding of versatile robots is intently subject to four vitals ideas for example discernment, restriction, cognition and movement control.

[3] The proposed framework comprises of portable rescue- robot's control Module. The venture proposes a versatile rescue robot which move in the calamity, quake tending zone and helps in distinguishing the live persons, harmed individuals, area and salvage framework activities.

[9]As live human being body discharge warm Radiations

,this is inwarded and controlled by the PIR sensor so as to be familiar with human .Once the individuals are found ,it quickly gives sound alarm, visual cautions to the specialists by way of the goal that help can make contact with the live individual so quick.

[15]Portable robots whether self-sufficient or tele- employable assume a significant job in various fields of human existence. Versatile robots are the most important part for working of researching zones in which human health is in danger of extinction. Police robots, fireman robots and rescue robots are instances of such submission.

[10] The commitment of this job is to give a sensor group to human identification in the urban calamity condition. The theory of the USAR venture at Carnegie Mellon is that the robot group ought to be minimal effort, semi-self- autonomous, heterogeneous, and cooperate underneath a human rescue team.

[5] This paper centers around two fundamental issues: how to precisely depict object structure at include level and how to fused different Local Structured Descriptors for part based model at topology level.

[11] The task proposes a self-governing mechanical vehicle that moves in the earthquake prone region what's more, helps in recognizing the live persons and rescue actions. Consequently valuable life can be spared by convenient identification in normal disasters indeed, even without the assistance of rescue team.

[4] This article has dissected the videotape and departing with ground explanation from the WTC robot-helped reaction. The investigation measured equally the conventional biological specialty of the human and robot specialists just as the societal specialty. While the information gathered was sparse because of the need to concentrate on the rescuing

The implemented framework comprises of a versatile rescue robot, RC control Module. The portable robot comprises of four units that are to be specific Motor driver unit, Camera unit, Micro-controller, Sensor unit, Motor driver part, Transmission unit. The sensor component should be legitimately interfaced to the Raspberry-pi. The sensor gadgets sends existing readings and send information to the Micro-controller. The controller circuit is answerable for transmitting this data. The RF module is interfaced with the raspberry pi using serial port. This information are refreshed by the PC/SERVER So that the rescue group can see the readings continuously and the camera is utilized to distinguish movement and live perception of the scenario.

\section{Methodology}




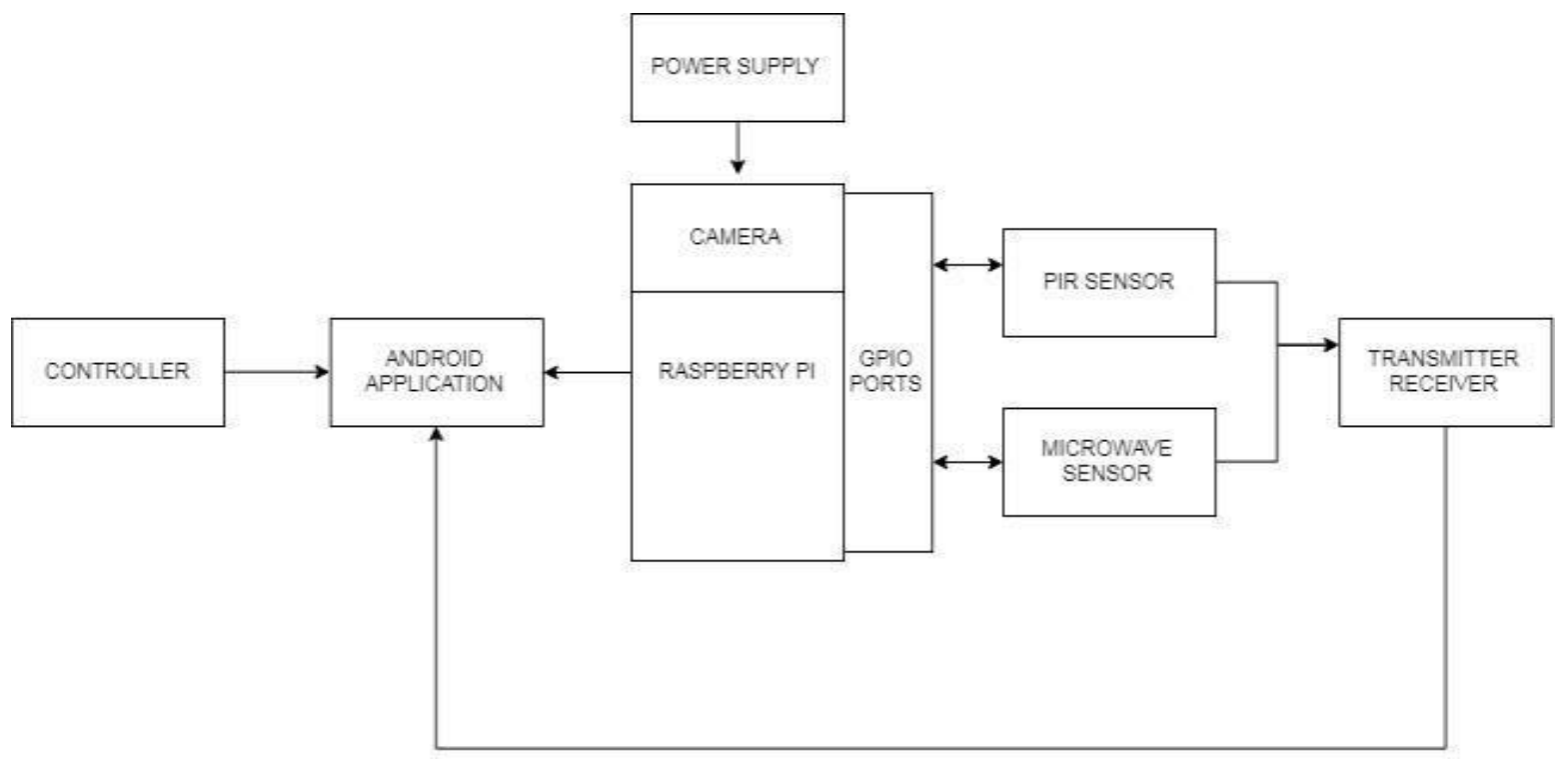

Figure 1. System architecture

We are using Raspberry PI or any other processor based boards. Physical radio controlled Bot which has long range enough to work with the Raspberry pi. As RC bot is required with radio controller which will help the bot to control its movements. As figure1 shows Android application will get the continuous data from Raspberry pi consisting sensors.

The camera will be used for continuous transmission of live imagery from the disaster prone area. The camera will be connected with the Raspberry pi. Using this imagery bot movement would be done. There are 2 PIR Sensors (large lens version) is used for the human detection and is mounted with Raspberry PI.

There are 2 HB100 10.525GHz Microwave Radar Sensors used for human motion detection which are trapped under the debris. As shown above microwave sensors will be connected with Raspberry PI sending data to android device. The Lithium Battery will give the required power to run the Raspberry pi.

The Rescue bot will be used in Rescue operations where it is difficult to find human life.

The Rescue bot will contain a frame which will secure the system components from any other fire or hazardous damage. The Rescue Bot will be connected to Mobile App for transmitting live feed from bot and sending any other notifications to the rescue team.

In implemented system we are going to take data from respective environment send it to rescue team's android device. The working system has been divided into the following modules.

- Module 1: Human detection using sensor

- Module 2: Live coverage from disaster area to android app.

- Module 3: Building physical Bot

Module 1:- Human being sensing encompass variety of technologies for detecting the company of a human body in an region of space. In this module we will detect human using different sensor in disaster area and send a detect human to the user or bot with the help of sensor are human movement can be detected. In PIR sensor are used to detect a human heat using that we identify a human presence Next is microwave sensor used to detect human motion or movement detect under the debris. The advantage of microwave sensor is it can detect only living thing not detect any non living thing movement.

Module 2:- The live coverage from camera and sensor data is streamed to android device. This can be achieved 
by using a apache webserver. the sensor data will be sent to a database followed with a webpage hosting the data. For streaming camera feed Mjpg streamer is used.

Module 3:- In this module we build a physical bot for to detect a human in disaster area in physical bot there are PIR, Microwave and camera sensor which are mounted on bot to detect a human.

Bot will be controlled by a radio control. Portability of the system will be achieved for porting whole system.

\section{A. Working of the System}

The UI made is accessible as Android Application. The Android Application is created utilizing Apache Cordova.
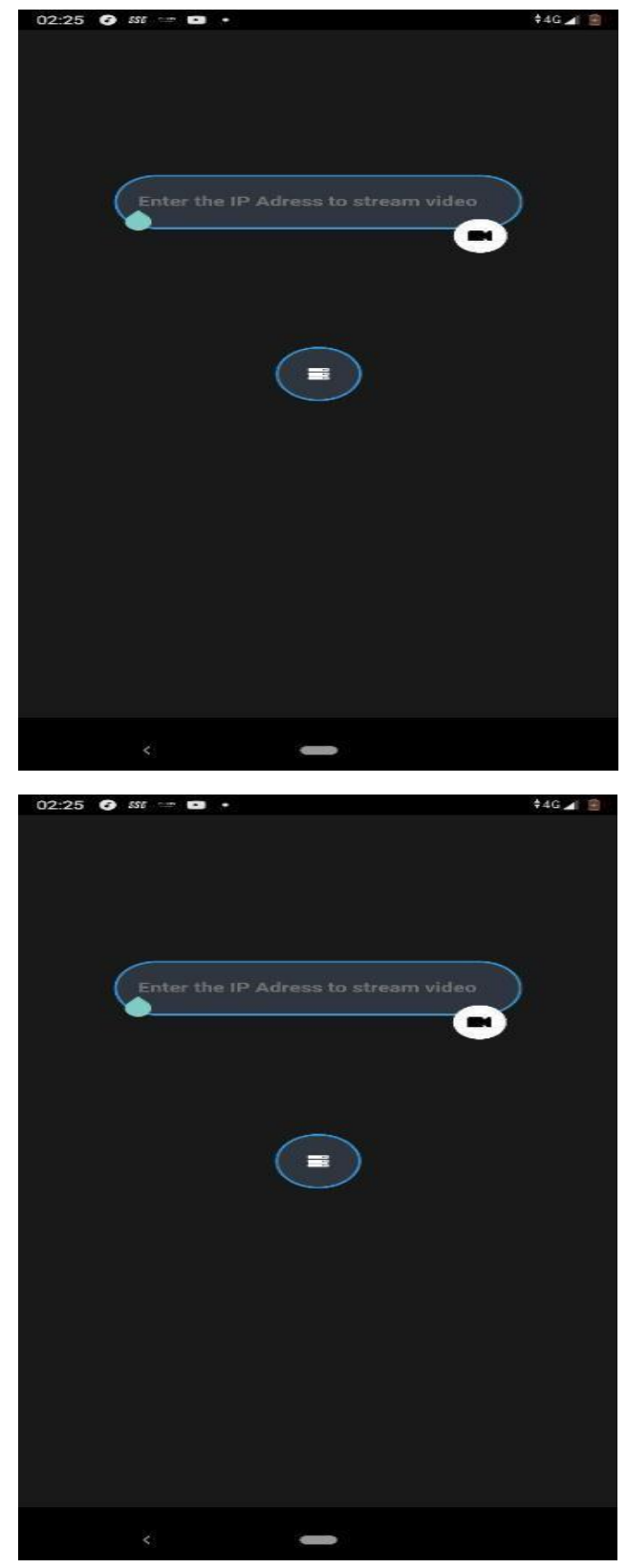


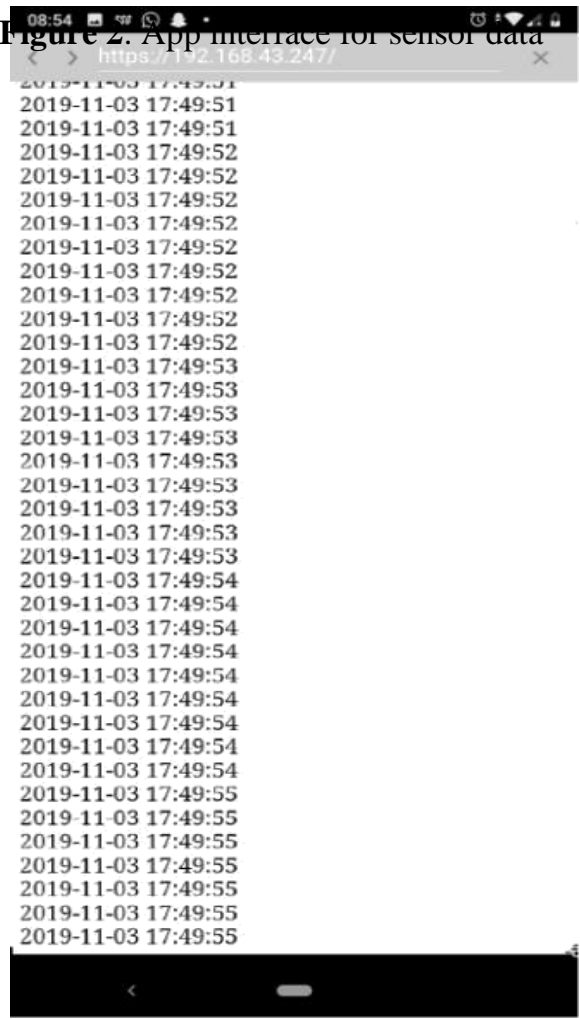

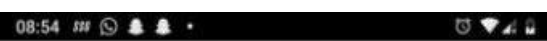
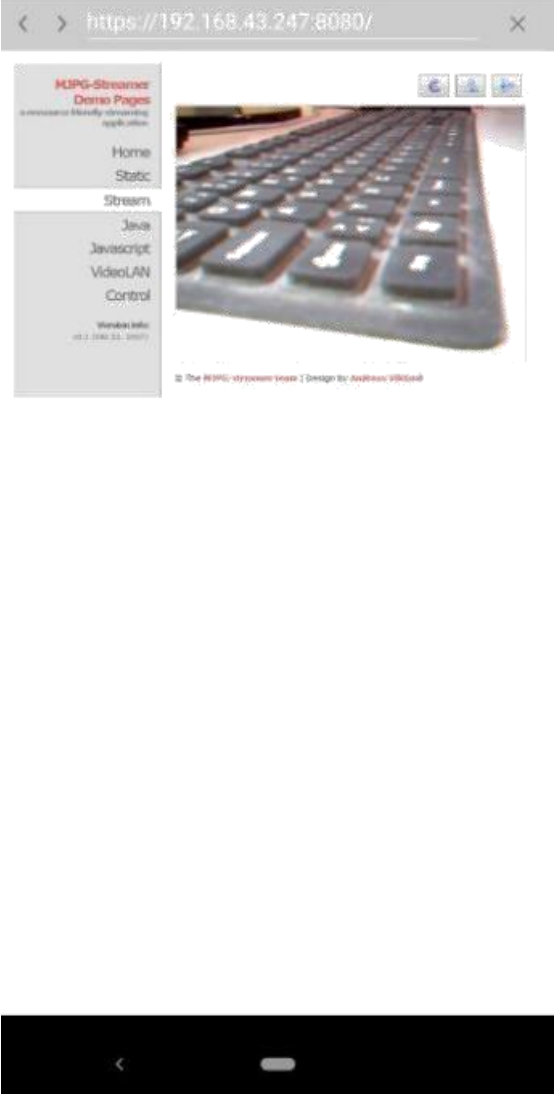

Figure 3. App interface for video streaming. 


\section{Working Principle}

The framework has two principle sides the transmitter side and the collector side. The transmitter side comprise of Raspberry Pi and sensors where's the beneficiary side consist of android controller. The PIR Sensor, Ultrasonic Sensor, Gas Sensor, Microwave Sensor are the contributions to Raspberry Pi. A web camera is likewise associated with Raspberry Pi to procure pictures and recordings in the event that of discovery. Raspberry Pi is associated with the Android application by making the remote network. Human can be identified utilizing PIR sensor, as it recognizes heat and human produce 9 - 10 microns of heat which can be recognized by PIR sensor. As the sensor's range is restricted it is mounted on vehicle with the goal that it can move around. Leveled out of a host raspberry pi the sensor radiates a short $40 \mathrm{KHz}$ blast. This blast adventures or goes through the air, hits a human and after that skips indeed to the sensor.

The collector side comprise of android application. By utilizing android application the robot can be worked. The yield of the sensors is gotten by android application and is shown on the application window. The photos/recordings caught by camera on robot are additionally seen on application window.

The hardware connections schematic diagram of equipment associations is shown in Fig. 2. pins are associated with the PIR module. Widespread coordinated and non-concurrent transmitter and receiver is utilized to keep communication between device along serial communication protocol. separately from the pins presently used in the circuit there is also a 5mp Rasp PI camera which is used for the image sensing and human detection. Other pins can be planned for controlling more numeral for other sensor to put into practice.

For the increasing figure of relays, some boards like Mega 2560 Arduino etc, can be worn with RPi by I2C communication protocol in a master-slave association. Mega 2560's have an edging over other accessible microcontrollers it contains 52 pins. It additionally has 3 serial ports that can be utilized to additionally broaden the length of the master-slave connection. A modest set-up with a case and two sensors are put on a door. The container contains RPi with RPi camera, Bluetooth module (HC05) and a Lithium ion bank (to control RPi). This case is mounted roughly at 8-10 feet of height. Sensors put on either sides of the entryway must have an ideal distance of 11.5 meter between each other.

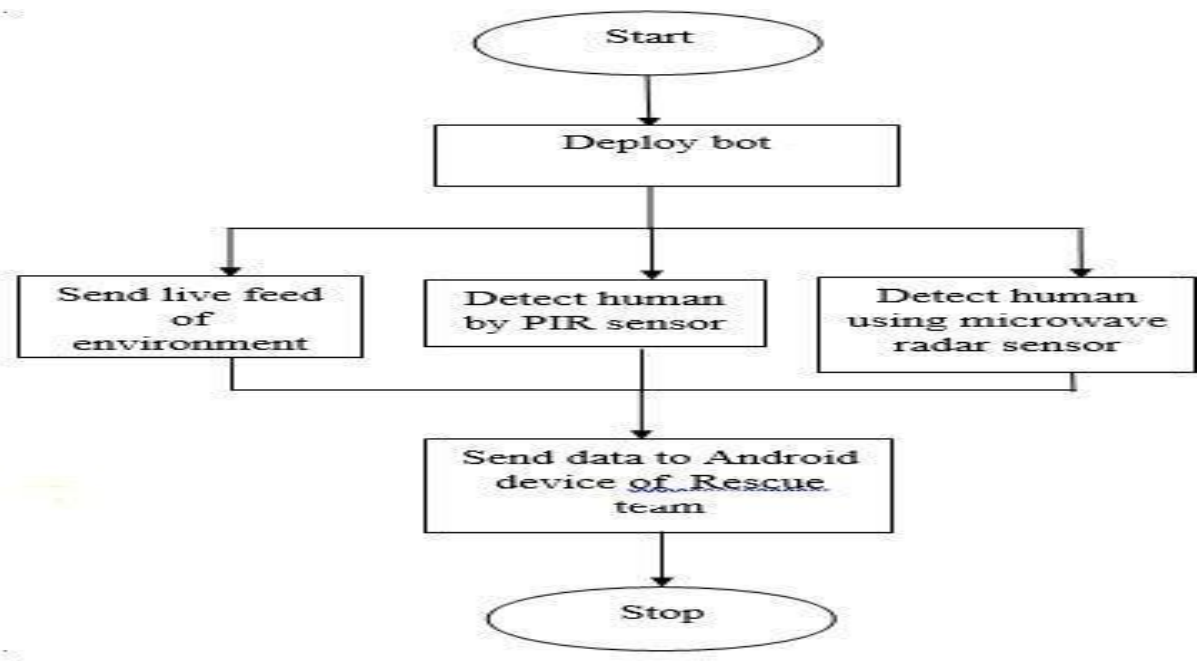

\section{Experimental Results}

Figure 4. Flow of the System

\section{A. Similar Method Analysis}

Table 1. Analysis of different methods

\begin{tabular}{|c|c|c|}
\hline METHOD & RESULT(\%) & YEAR \\
\hline$[15]$ & $80.06 \%$ & 2006 \\
\hline
\end{tabular}




\begin{tabular}{|c|c|c|}
\hline$[14]$ & $84.70 \%$ & 2014 \\
\hline$[9]$ & $88.56 \%$ & 2016 \\
\hline Proposed System & $92.32 \%$ & $2019-20$ \\
\hline
\end{tabular}

The chief novelty implicated is amalgamation of multiple use of sensor in human detection that is previously discussed in introduction. The GUI is formed for live streaming of the video feed coming from the BOT this also helps in to drive the robot. To have a simpler hardware interaction with the robot, it would be constructive to attach the infrared camera. Our methodology is that a robot should have a complete display of the earth, meld the data from the majority of its sensors, the client would then be able to choose the commands and advise the robot where it should move. The user interface for the sensors must sustain following instructions.

Among from the previous innovations we found some causalities and worked on it we found the above results.

\section{Conclusion and Future Work}

This paper depicts a semi-autonomous robot plan, produced for urban search and rescue tasks, in particular its mechanical structure, human detecting abilities and correspondences, administrator interface and programming design. The robot utilizes remote interchanges, with a possibility for fastened task. The tie conveys power \& interchanges, with a passageway on end. Assembling and disassembling the robot is practiced remotely by the administrator with the assistance of a camera situated inside the robot, and speaks to the most inventive element of bot. Major conclusions can be obtained from this proposed system is that: This system can be used in rescue operations as well as in covert missions of forces The system implemented on a bot can also be implemented on drone technology in order to scan huge amount of area. Further improvement should be possible by utilizing a high end camera to build caliber, clarity \& nature of pictures caught. Future visualized work concerns about the flexible self-governance capacities, both for the single-robots and to multi-robot groups, in order for to liberate the administrator from most monotonous work, and for to support finding unfortunate casualties and driving the robot satisfactorily.

\section{References}

1. Rupnar Pallavi Sharad, Prof Thorat P.R"Live Human Detecting Robot for Earthquake Rescue Operation " Systems, Man and Cybernetics, Part A: Systems and Humans, IEEE Transactions, vol. 41.1, 2011, pp. 24-33.

2. Shubham Mathur Balaji Subramanian, Sanyam Jain, Kajal Choudhary. "Human detector and controller using Raspberry pi controller." Computer Vision and Pattern Recognition-Workshops, 2005. CVPR Workshops. IEEE Computer Society Conference o. IEEE, 2005.

3. Alive human body detection system using an autonomous mobile rescue robot India Conference (INDICON), 2011 Annual IEEE.

4. J. Casper "Human-Robot Interactions during the Robot-Assisted Urban Search and Rescue Response at The World Trade Center", MS Thesis, Computer Science and Engineering, USF, South Florida, 2002.

5. Zhang, Junge, et al. "Boosted local structured hog-lbp for object localization." Computer Vision and Pattern Recognition (CVPR), 2011 IEEE Conference on. IEEE, 2011.

6. Bradski, Gary, and Adrian Kaehler. Learning OpenCV: Computer vision with the OpenCV library. "O'Reilly Media, Inc.", 2008.

7. Rauter, Michael. "Reliable human detection and tracking in top-view depth images." Proceedings of the IEEE Conference on Computer Vision and Pattern Recognition Workshops. 2013.

8. Carloso Marques, Pedrot Lima, Rodrigoy Ventura "Semiautonomous robot for rescue operations"@International Conference on Intelligent Robots and Systems, October 9-15,2006, Beijing, China.

9. Kalyani Singh, Isha Chaudhary, Archana Kumari, Bhojraj Singh, of India entitled "Human live detection robot used in natural disasters" published in ISSN 23213361 C 2016 IJESC. 
10. Leslie Burion, Steve (2004), Casper, J. (2002), Amerada, Yams, Igarashit, \&Matsunos, (2004) and Cappello, Cetal (2005) "Human Detection for Robotic Urban Search and Rescue" Carnegie Mellon University, Institute of Production Robotique(IPR)LSRO2

11. VRAI-Group, Microtechnique, February 2004 (Mr.S.PVijayaragavan, Hardeep Pal Sharma, Gunasekar.C.H, S.Adithya Kumar, Live Human Detecting Robot for Earthquake Rescue Operation, International Journal of Business Intelligents, ISSN: 2278-2400

12. Dnyanoba, Birajdar, Amar, B. Nagajayanthi, and Prakash Ramachandran. "Development of an Embedded System to Track the Movement of Bluetooth Devices based on RSSI." "Indian Journal of Science and Technology", vol. 8.19, 2015.

13. Adel Aneiba, Khaled Hormos, of Libya entitled "A Model for Remote Controlled Mobile Robotic over Wi-Fi Network Using Arduino Technology" Published in Nov. 2014 as 978-1-78561-072-1/( 2014 IEEE.

14. S. Ali A. Moosavian, Hesam Semsarilar Arash Kalantari of bejing China entitled "Design and manufacturing of a mobile rescue robot" published in 2006 as 1-4244-0259-X/06/@ 2006 IEEE. 